\title{
System Support and Applications for Mobile Computing - Extended Abstract -
}

\author{
Norbert Diehl, Albert Held \\ Daimler-Benz AG, Research Center Ulm, Information Technology \\ Wilhelm-Runge-Straße 11, P.O. Box 2360, 89013 Ulm, Germany \\ Phone: +49 731505 2132/2831, Fax: + 497315054218 \\ e-mail: \{diehl, held\}@dbag.ulm.DaimlerBenz.COM
}

\section{INTRODUCTION: DISTRIBUTED, MOBILE APPLICATIONS}

In future networks, mobile computers will become increasingly important. New portable computers and wireless communications technologies particularly enable mobile computing. Distributed, mobile applications allow information access anywhere, anytime. Mobility (in the sense of location independence) and adequate information support are no longer opposites but are supported simultaneously.

The possibility to support mobility and thus changing locations (i.e. network access points) while being connected offers new possibilities for future applications, but also introduces new problems. Some of them are:

- A basic but far-reaching fact is that the users are moving and thus the topology of the system is constantly changing. This also includes location dependent information and therefore the need for new addressing schemes.

- The mobile terminals will always be less powerful than the stationary computers. Thus we have a performance disparity between the mobile and stationary computers, that has a strong influence on the system design, e.g., workload balance, etc.

- The data rates of wireless connections will always be clearly lower than those of wired connections. Wireless connections are not as reliable as wired. There is also the need to support interworking in heterogeneous networks.

Additional problems due to mobility and the specific characteristics of portable computers and wireless communications are dynamic configuration, moving resources, reachability, data and function consistency. All these problems have to be treated in more than one of the communication layers. A full system architecture is necessary. This includes an additional "mobility layer" above the OSI transport layer.

We present a system called MOBI-DICK that addresses the requirements of mobile computing and we discuss two fields of applications in more detail: mobile services in the industrial environment and traffic-telematics.

\section{MOBI-DICK}

MOBI-DICK is a system platform to support distributed mobile applications and addresses basic aspects of mobile computing:

- terminal mobility -user mobility - service mobility

- location dependent services - location independent services

- ad-hoc networking 
- autonomous work during disconnection

- IT-security (authentication, authorization, ...)

- adaption and support of differnet wireless and wired communication systems

MOBI-DICK includes an application manager for mobile applications and a service directory and service trading mechanisms that allow location transparency and location awareness. Basic security services for authentication and access control are integrated in MOBI-DICK

\section{MOBILE APPLICATIONS}

\section{Mobile Services in the Industrial Environment}

Mobile applications in industrial environments add special requirements. Typical scenarios:

- plant operatives are mobile: For example, the set-up or maintenance staff, equipped with mobile terminals, is working at different locations and having full multi-media information support .

- products are mobile: Within a large production plant the products, e.g. cars, are moved from one manufacturing location to another. To allow the easy identification of the products and the concrete work to be done as well as the status of work carried out, the product should be equipped with escort memory systems (ems) that can be read and written via wireless communication.

- machinery or production systems are mobile: Within h ghly flexible production plants the machinery may be reconfigured and moved relatively often. Problems such as recabling, installing and interconnecting new sensors and control units have to be considered.

- transportation systems or platforms in a manufacturing plant are mobile: To achieve an optimal material flow, transportation systems should be equipped with sufficient computing facilities and access to the plant's communication network.

For all types, wireless communications and new multi-media mobility servicesare needed. Thus we will both look at the requirements for the new high performance radio network and the application support services for mobility, multi-media, and interworking.

\section{Traffic Telematics}

Traffic Telematic Services such as Guidance-Systems, Tourist- and Travel-Information, Traffic-Information, Trip-Planning, Fleet Management, Mobile Office, etc. become more and more important. These services are used from stationary hosts (e.g., PC at home) as well as from mobile hosts (in vehicles).

In addition to global service providers, a large number of smaller service providers with local services (e.g., hotel reservation, local entertainment) or specialized services (e.g., stock market information) will coexist. This leads to dynamic systems with various distributed information sources. While driving from one city to another, several service providers are involved. A seamless service handover with adopted service trading protocols is needed.

Other topics of interest are: flexibility and scalability of the service system, efficient information acquisition, filtering and presentation, optimized use of the available communication systems, location dependency.

We show how these requirement can be handled and present solutions based on the MOBIDICK system. 